TRANSACTIONS OF THE

AMERICAN MATHEMATICAL SOCIETY

Volume 359, Number 9, September 2007, Pages 4285-4295

S 0002-9947(07)04161-X

Article electronically published on March 20, 2007

\title{
THE ELEMENTARY TRANSFORMATION OF VECTOR BUNDLES ON REGULAR SCHEMES
}

\author{
TAKURO ABE
}

\begin{abstract}
We give a generalized definition of an elementary transformation of vector bundles on regular schemes by using Maximal Cohen-Macaulay sheaves on divisors. This definition is a natural extension of that given by Maruyama, and has a connection with that given by Sumihiro. By this elementary transformation, we can construct, up to tensoring line bundles, all vector bundles from trivial bundles on nonsingular quasi-projective varieties over an algebraically closed field. Moreover, we give an application of this theory to reflexive sheaves.
\end{abstract}

\section{INTRODUCTION}

An elementary transformation is the theory on ruled surfaces, which enables us to construct a new ruled surface from one given through birational geometry. In Mar], Maruyama generalized this method to apply to the construction theory of vector bundles. By using his idea and theory, we can construct a lot of indecomposable vector bundles on schemes, especially those on low dimensional projective varieties. On the other hand in $\mathrm{Su}-2$ and $\mathrm{Su}-3$, Sumihiro gave an another definition of an elementary transformation of vector bundles on schemes, which is closely related to the geometric characterization of the elementary transformation of ruled surfaces. Let us review them shortly.

The definition of an elementary transformation given by Maruyama is, roughly speaking, to give a surjection from the given vector bundle to the vector bundle whose support is a divisor. This is very useful to construct vector bundles and has a lot of applications and examples. However, on higher dimensional varieties, there is a disadvantage that not all vector bundles can be constructed from trivial bundles by this method.

The definition given by Sumihiro can be applied to the vector bundle construction on higher dimensional varieties, i.e., by using his theory, we can construct, up to tensoring line bundles, all vector bundles on any dimensional nonsingular quasi-projective varieties over an algebraically closed field from trivial bundles. To apply this elementary transformation, we need some geometric data. Note that the explicit relation between these two was not clear.

In this article, we give a new definition of an elementary transformation of vector bundles on regular schemes by using Maximal Cohen-Macaulay sheaves on their divisors. This is a natural generalization of Maruyama's definition, and in the special case, it can be interpreted into Sumihiro's definition, i.e., by this theory,

Received by the editors July 16, 2004 and, in revised form, July 23, 2005.

2000 Mathematics Subject Classification. Primary 14F05.

(c) 2007 American Mathematical Society Reverts to public domain 28 years from publication 
we can make it clear how the two definitions of an elementary transformation are related. Also, this definition overcomes the disadvantage of the former definition when referring to higher dimensional cases. Moreover, from this new definition, we can obtain several results not only on vector bundles but also on reflexive sheaves, by using torsion free sheaves on divisors. Consequently, we can construct, up to tensoring line bundles, all the vector bundles and reflexive sheaves from trivial bundles on nonsingular quasi-projective varieties over an algebraically closed field of any characteristic. These are main results in this article and described in Theorem 1.3 and Theorem 2.5.

Contents of this article are as follows.

In section one, we give a new definition of an elementary transformation by introducing the concept of ET-data $(Z, F, \varphi)$. The main result is Theorem 1.3 as mentioned above. The relation between the definition given in this section and Maruyama's is described after the proof of Theorem 1.3. and that between ours and Sumihiro's in Proposition 1.5.

In section two, we apply the new elementary transformation to reflexive sheaves. In the same meaning, we can construct all reflexive sheaves from trivial bundles by this method (Theorem 2.5).

Notation. We denote by $\mathbb{Z}$ the integer ring and by $\mathbb{C}$ a complex number field. For a commutative ring $A, M(r, A)$ is the set of $r \times r$ matrices with entries in $A$. $I_{r} \in M(r, A)$ is a unit matrix.

In this article, the term "variety" means an integral algebraic scheme over a field. We use the terms "vector bundle" and "locally free sheaf" interchangeably.

Let us assume that $X$ is a Noetherian scheme and $F$ is a coherent sheaf on $X$. By $\operatorname{Supp}_{X}(F)$ or $\operatorname{Supp}(F)$, we denote the support of $F$ as an $\mathcal{O}_{X}$-module. By $\operatorname{Ass}_{X}(F)$, we denote the associated points of $F$ as an $\mathcal{O}_{X}$-module. $\operatorname{Sing}_{X}(F)$ or $\operatorname{Sing}(F)$ is the set of points $x \in X$ at which $F_{x}$ is not a free $\mathcal{O}_{x, X}$-module. $F^{*}$ is the dual sheaf of $F$, i.e., $F^{*} \simeq \mathcal{H o m}_{X}\left(F, \mathcal{O}_{X}\right)$.

\section{Generalized elementary transformation}

We give a definition of a generalized elementary transformation of vector bundles. First, let us review the definition of Maximal Cohen Macaulay sheaves. We say a coherent sheaf $F$ on a Noetherian scheme $X$ is a Maximal Cohen-Macaulay $\mathcal{O}_{X^{-}}$ module if

$$
\operatorname{depth}_{\mathcal{O}_{x, X}}\left(F_{x}\right)=\operatorname{dim} \mathcal{O}_{x, X} \text { for all } x \in X .
$$

"Maximal Cohen-Macaulay" is often denoted by "MCM" in this article. Secondly, we define the data which play the key role in the generalization of elementary transformations.

Definition 1.1. Let $X$ be a regular scheme, $E$ be a vector bundle of rank $r(\geq 2)$ on $X$, and $m$ be an integer such that $1 \leq m \leq r-1$. We say the triple $(Z, F, \varphi)$ is $m$ elementary-transformation-data for $E$ ( $m$-ET-data for $E$, for short) if the following three conditions are satisfied:

1) $Z$ is an effective reduced divisor on $X$,

2) $F$ is an MCM $\mathcal{O}_{Z}$-module of rank $r-m$, and

3) $\varphi$ is a surjection from $E$ onto $F$ as $\mathcal{O}_{X}$-modules. 
When there is no confusion, we often omit the surjection $\varphi$ of $\operatorname{ET}$-data $(Z, F, \varphi)$ and denote it by $(Z, F)$. Using this concept, we can define the generalized elementary transformation of vector bundles as follows:

Definition 1.2. With the notation in Definition 1.1, we denote the kernel of $\varphi$ : $E \rightarrow F$ by $\operatorname{elem}_{F}(E)$ and call it an elementary transformation of $E$ by $(m-)$ ET-data $(Z, F)$.

The following lemma is easy to see from the argument on the depth and projective dimension, combined with the Auslander-Buchsbaum formula.

Lemma 1.1. If $(Z, F)$ is ET-data for a vector bundle $E$ of rank $r$, then $\operatorname{elem}_{F}(E)$ is also a vector bundle of rank $r$.

By Lemma 1.1, this elementary transformation becomes a method to make a new vector bundle from a given one. In Maruyama's definition of an elementary transformation $\mathrm{Mar}$, the above $F$ is not MCM but a vector bundle on $Z$. As we saw in the introduction, his definition and results cannot be sufficiently applied to the higher dimensional cases. In Definition 1.1, we simply extend his definition to get a stronger result on higher dimensional cases.

Before some basic properties of the elementary transformation and the main theorem, let us see some examples of elementary transformations, not only of ours but also of Maruyama's.

Example. (1) The most famous and traditional example of the elementary transformation is that of ruled surfaces. A ruled surface $S$ on some smooth projective curve $C$ over an algebraically closed field, which is a $\mathbf{P}^{1}$-fiber bundle on $C$ through the projection $\pi: S \rightarrow C$, corresponds to some rank two vector bundle $E$ on $C$, i.e., $S \simeq \mathbf{P}(E)$ over $C$. Take a point $x \in C$ and consider the canonical surjection:

$$
E \rightarrow k(x) \rightarrow 0
$$

where $k(x)$ is a skyscraper sheaf at $x . k(x)$ is a locally free sheaf on a divisor $\{x\}=\operatorname{Spec}(k(x))$, so this is Maruyama's elementary transformation. Its kernel $E^{\prime}:=\operatorname{elem}_{k(x)}(E)$ is also a rank two vector bundle, and gives a new ruled surface $\mathbf{P}\left(E^{\prime}\right)$ over $C$. It is known that $\mathbf{P}\left(E^{\prime}\right)$ can be obtained by the following geometric way: Take some point $y \in \pi^{-1}(x)$ and blow up $S$ with the center $y$ and get the blow-up variety $B$. Then we can contract the original fiber $\pi^{-1}(x)$ of $B$ by Castelnuovo's theorem, and the variety constructed after this blow-down is just $\mathbf{P}\left(E^{\prime}\right)$. For details, see Mar.

(2) Consider a hyperplane arrangement $\mathcal{A}$ in $\mathbf{P}_{\mathbb{C}}^{n}(n \geq 2)$, i.e., the set $\mathcal{A}=$ $\left\{H_{1}, \cdots, H_{l} \mid H_{1}, \cdots, H_{l}\right.$ is a hyperplane in $\mathbf{P}_{\mathbb{C}}^{n}$ and $H_{i} \neq H_{j}$ if $\left.i \neq j\right\}$. We assume that $\operatorname{codim}_{\mathbf{P}_{\mathbb{C}}^{n}}\left(H_{i_{1}} \cap \cdots \cap H_{i_{s}}\right)=s$ for all $H_{i_{j}} \in \mathcal{A}$ (such an arrangement is called generic). Put a divisor $Z:=\bigcup_{i=1}^{l} H_{i}$, and take a point $x \in \mathbf{P}_{\mathbb{C}}^{n}$ and a meromorphic $q$-form $\omega$ around $x$ which may have poles only along $Z$. I.e., we say $\omega$ is a logarithmic $q$-form along $Z$ if $h \omega$ and $(d \omega) h$ are holomorphic, where $h$ is a local equation of $Z$ at $x$. By using this concept, K. Saito defined a coherent sheaf $\Omega_{\mathbf{P}_{\mathbb{C}}^{n}}^{q}(\log (\mathcal{A}))$ of rank $n$ on $\mathbf{P}_{\mathbb{C}}^{n}$ by 
the following manner $\underline{\mathrm{Sa}}$ :

$$
\begin{aligned}
\Omega_{\mathbf{P}_{\mathbb{C}}^{n}}^{q}(\log (\mathcal{A}))_{x} & :=\text { germ of logarithmic } q \text {-form at } x\}, \text { and } \\
\Omega_{\mathbf{P}_{\mathbb{C}}^{n}}^{q}(\log (\mathcal{A})) & :=\bigcup_{x \in \mathbf{P}_{\mathbb{C}}^{n}} \Omega_{\mathbf{P}_{\mathbb{C}}^{n}}^{q}(\log (\mathcal{A}))_{x} .
\end{aligned}
$$

For a generic $\mathcal{A}, \Omega_{\mathbf{P}_{\mathbb{C}}^{n}}^{1}(\log (\mathcal{A}))$ becomes locally free of rank $n$. We can construct this "Logarithmic differential sheaf" by the following elementary transformation [DK]:

$$
0 \rightarrow \Omega_{\mathbf{P}_{\mathbb{C}}^{n}}^{1} \rightarrow \Omega_{\mathbf{P}_{\mathbb{C}}^{n}}^{1}(\log (\mathcal{A})) \rightarrow \bigoplus_{i=1}^{l} \mathcal{O}_{H_{i}} \rightarrow 0
$$

This exact sequence plays an important role in finding the jumping line, or in investigating the stability of $\Omega_{\mathbf{P}_{\mathbb{C}}^{n}}^{1}(\log (\mathcal{A}))$ in terms of combinatorics of arrangements in $[\mathrm{DK}$.

(3) In [Ta-1], Tango gave a nonsplitting rank two vector bundle on $\mathbf{P}_{\mathbb{K}}^{5}$ ( $\mathbb{K}$ is an algebraically closed field of characteristic two). On any algebraically closed field $\mathbb{K}$, no other rank two indecomposable bundle on $\mathbf{P}_{\mathbb{K}}^{5}$ is known so far. We can give the explicit data to construct it from trivial bundles by the elementary transformation. They can be seen in section five of [A], and this can be done by the elementary transformation in Definition 1.1.

The elementary transformation satisfies some functorial properties as follows.

Proposition 1.2. Let $X, X^{\prime}$ be regular schemes, $E_{i}$ be a vector bundle on $X$ of rank $r_{i}(i=1,2)$, and $\left(Z, F_{i}\right)$ be an $m_{i}$-ET-data for $E_{i}(i=1,2)$. Then the following holds:

1) Let $f: X^{\prime} \rightarrow X$ be a morphism such that $\left(f^{*} Z, f^{*} F_{1}\right)$ is also $m_{1}$-ET-data for $f^{*} E_{1}$. Then $f^{*}\left(\operatorname{elem}_{F_{1}}\left(E_{1}\right)\right) \simeq \operatorname{elem}_{f^{*} F_{1}}\left(f^{*} E_{1}\right)$.

2) If $L$ is a line bundle on $X$, then it holds that

$$
\operatorname{elem}_{F_{1}}\left(E_{1}\right) \otimes L \simeq \operatorname{elem}_{F_{1} \otimes L}\left(E_{1} \otimes L\right) .
$$

3) $\operatorname{elem}_{F_{1}}\left(E_{1}\right) \oplus \operatorname{elem}_{F_{2}}\left(E_{2}\right) \simeq \operatorname{elem}_{F_{1} \oplus F_{2}}\left(E_{1} \oplus E_{2}\right)$.

4) Assume that $m_{1}=m_{2}=m$ and $r_{1}=r_{2}=r$. If there exist isomorphisms $h$ : $E_{1} \rightarrow E_{2}$ and $\bar{h}: F_{1} \rightarrow F_{2}$ which makes the following diagram commutative:

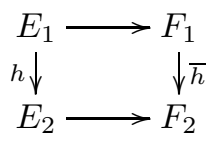

then it holds that $\operatorname{elem}_{F_{1}}\left(E_{1}\right) \simeq \operatorname{elem}_{F_{2}}\left(E_{2}\right)$.

5) Let $(Z, F, \varphi)\left(\operatorname{resp}:\left(Z^{\prime}, F^{\prime}, \varphi^{\prime}\right)\right)$ be $m$ (resp : $\left.m^{\prime}\right)$-ET-data for a vector bundle $E$ of rank $r$ on $X$. Let us put $\operatorname{elem}_{F}(E)=: G$, $\operatorname{elem}_{F^{\prime}}(E)=: G^{\prime}$, and $f: G \rightarrow E, f^{\prime}: G^{\prime} \rightarrow E$. If $\varphi \circ f^{\prime}\left(\right.$ or $\left.\varphi^{\prime} \circ f\right)$ is surjective, then we can define $\operatorname{elem}_{F}\left(\operatorname{elem}_{F^{\prime}}(E)\right)$ and $\operatorname{elem}_{F^{\prime}}\left(\operatorname{elem}_{F}(E)\right)$. Moreover, they are isomorphic.

Proof. 1), 2), 3) and 4) are easy to show, so we left them to the reader. We prove $5)$. We may assume that $\varphi^{\prime} \circ f$ is surjective. Then we have the following exact 
sequences:

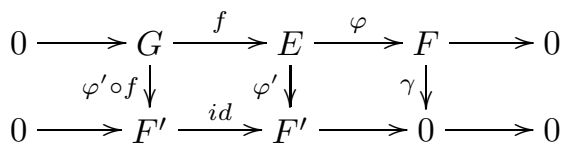

Then by the snake lemma, we have

$$
\operatorname{ker}\left(\varphi^{\prime}\right) \rightarrow \operatorname{ker}(\gamma) \rightarrow \operatorname{coker}\left(\varphi^{\prime} \circ f\right) \rightarrow 0,
$$

which is equivalent to the surjection

$$
E^{\prime} \stackrel{\varphi \circ f^{\prime}}{\rightarrow} F \rightarrow 0
$$

Hence we can define $\operatorname{elem}_{F^{\prime}}\left(\operatorname{elem}_{F}(E)\right)$ and $\operatorname{elem}_{F}\left(\operatorname{elem}_{F^{\prime}}(E)\right)$. Moreover, we have

$$
\begin{aligned}
\operatorname{elem}_{F^{\prime}}\left(\operatorname{elem}_{F}(E)\right) & \simeq \operatorname{ker}\left(\varphi^{\prime} \circ f\right) \simeq G \cap \operatorname{ker}\left(\varphi^{\prime}\right) \simeq G \cap G^{\prime} \\
& \simeq G^{\prime} \cap \operatorname{ker}(\varphi) \simeq \operatorname{ker}\left(\varphi \circ f^{\prime}\right) \simeq \operatorname{elem}_{F}\left(\operatorname{elem}_{F^{\prime}}(E)\right)
\end{aligned}
$$

as desired.

Now, we state the main theorem, which enables us to construct all vector bundles from a direct sum of trivial bundles.

Theorem 1.3. Let $X$ be a nonsingular quasi-projective variety over an algebraically closed field $\mathbb{K}, \mathcal{O}_{X}(1)$ be an ample line bundle on $X$, and $E$ be a vector bundle of rank $r(>1)$ on $X$. Then there exist 1-ET-data $(Z, F)$ for $\mathcal{O}_{X}^{r}$ such that $Z$ is normal and $\operatorname{elem}_{F}\left(\mathcal{O}_{X}^{r}\right) \simeq E \otimes L$ for some line bundle $L \in \operatorname{Pic}(X)$. Moreover, when $\operatorname{dim} X \geq 2$, we can take $Z$ as an integral divisor.

Proof. Tensoring $\mathcal{O}_{X}(d)$ for $d \gg 0$ and using 2) of Proposition 1.2, we may assume that $E$ is very ample. Let us put the projective bundle $\mathbf{P}(E)$ over $X$ associated to $E$ and put the canonical projection $\pi: \mathbf{P}(E) \rightarrow X$. Then there are global sections $s_{1}, \cdots, s_{r} \in H^{0}(X, E)$ such that for the divisors $D_{i}(i=1, \cdots, r)$ in $\mathbf{P}(E)$ defined by the section $s_{i}$, the intersection $D_{1} \cap \cdots \cap D_{r}$ is a smooth subscheme of pure codimension $r$ in $\mathbf{P}(E)$. Let us put

$$
\begin{aligned}
Z & :=Z\left(s_{1} \wedge \cdots \wedge s_{r}\right), \\
W_{i} & :=Z\left(s_{1} \wedge \cdots \wedge s_{i-1} \wedge s_{i+1} \wedge \cdots \wedge s_{r}\right)(i=1, \cdots, r), \\
B & :=\bigcap_{i=1}^{r} W_{i} \\
U & :=Z \backslash B .
\end{aligned}
$$

From the calculation of its Jacobian, we can see that $Z \cap U$ is a smooth divisor of $U$ and $\operatorname{Sing}(Z)=B$. If $B$ contains a point whose codimension in $Z$ is one, then since $\operatorname{dim} \pi^{-1}(x) \geq 1$ for all $x \in B$, it holds that $\operatorname{dim} \pi^{-1}(B) \geq \operatorname{dim} Z$. This is a contradiction. Hence all the points which belong to $B$ have codimensions greater than or equal to two. This implies that $Z$ is regular in codimension one. Moreover, since $Z$ is Cohen-Macaulay, it satisfies Serre's criterion for normality. Hence $Z$ is normal. When $\operatorname{dim} X \geq 2$, it follows that $Z \cap U \neq \phi$, hence it is a nonempty smooth divisor in $U \neq \phi$. So in this case, $Z$ is integral.

Now, let us see the exact sequence

$$
\mathcal{O}_{X}^{r} \stackrel{\left(s_{1}, \cdots, s_{r}\right)}{\rightarrow} E \rightarrow F \rightarrow 0
$$


which is induced by the sections $s_{1}, \cdots, s_{r}$. Take and fix a point $x \in X$, put $e_{1}, \cdots, e_{r}$ as the free basis of $\mathcal{O}_{x, X}^{r}$, and $X_{1}, \cdots, X_{r}$ as the free basis of $E_{x}$. For $i=1, \cdots, r$, write $\left(s_{i}\right)_{x}=\left(s_{i 1}, \cdots, s_{i r}\right)$ by this basis. Then the morphism $\mathcal{O}_{X}^{r} \rightarrow E$ at $x$ is a matrix ${ }^{t} S={ }^{t}\left(s_{i j}\right)_{i, j=1}^{r}$. If we denote by $Y_{i}$ the image ${ }^{t} S\left(e_{i}\right)$, it holds that

$$
Y_{i}=s_{i 1} X_{1}+\cdots+s_{i r} X_{r}(i=1, \cdots, r) .
$$

So locally $F=\bigoplus A X_{i} / \sum A Y_{i}$, and $F=0$ if and only if there exists a matrix $T \in M\left(r, \mathcal{O}_{x, X}\right)$ such that $T S=S T=I_{r}$. Such $T$ exists if and only if $s=\operatorname{det} S$ is a unit at $x$. This implies $\operatorname{Supp}_{X}(F)=Z$ and $F$ is an $\mathcal{O}_{Z}$-module. Next, we show that $F$ is an MCM sheaf on $Z$ of rank 1 . Note that $s$ is a local equation of $Z=Z\left(s_{1} \wedge \cdots \wedge s_{r}\right)$ at $x$ and $\operatorname{det}(S)=s$. Hence if we denote the adjoint matrix of $S$ by $S^{\prime}$, it holds that $S S^{\prime}=S^{\prime} S=s I_{r}$. Combining these observations with the following lemma, we can see $F$ is MCM.

Lemma 1.4. Let $A$ be a Cohen-Macaulay Noetherian local ring, $r(>1)$ be an integer, and $S, S^{\prime} \in M(r, A)$ be $r \times r$ matrices such that $S S^{\prime}=S^{\prime} S=s I_{r}$ for some nonzero-divisor $s \in A \backslash A^{\times}$. Put $A / s A=: \bar{A}$ and denote the image of $a \in A$ in $\bar{A}$ by $\bar{a}$. Assume that $\operatorname{rank} \bar{S} \neq 0$ and $\operatorname{rank} \overline{S^{\prime}} \neq 0$. Then $M:=\operatorname{coker}\left({ }^{t} S: A^{r} \rightarrow A^{r}\right)$ is a Maximal Cohen-Macaulay $\bar{A}$-module.

Proof of lemma. Put $\operatorname{dim} A=n \geq 1$. Clearly, $A$ and $\bar{A}$ are Cohen-Macaulay local rings. Hence there is a regular sequence

$$
s=z_{1}, z_{2}, \cdots, z_{n}
$$

for $A$. We shall prove that

$$
\overline{z_{2}}, \cdots, \overline{z_{n}}
$$

is a regular sequence for $M$ by induction on its length. Assume that $\left(\overline{z_{2}}, \cdots, \overline{z_{l-1}}\right)$ is a regular sequence for $M$ (where $2 \leq l \leq n+1$ ). Take

$$
\sum_{i=1}^{r} \overline{a_{i}} \overline{X_{i}} \in M
$$

where $\left\{X_{i}\right\}_{i=1}^{r}$ is the free basis of $A^{r}$, and assume that

$$
\overline{z_{l}} \sum \overline{a_{i}} \overline{X_{i}}=0 \text { in } M /\left(\overline{z_{2}}, \cdots, \overline{z_{l-1}}\right) M .
$$

Then there are elements $b_{i}, c_{k i} \in A$ such that

$$
z_{l} \sum_{i=1}^{r} a_{i} X_{i}=\sum_{i=1}^{r} b_{i} Y_{i}+\sum_{i=1}^{r} \sum_{k=2}^{l-1} c_{k i} z_{k} X_{i}
$$

where $S=\left(s_{i j}\right)$ and $Y_{i}=\sum_{j=1}^{r} s_{i j} X_{j}$. Then for all $j=1, \cdots, r$, we have

$$
z_{l} a_{j}=\sum_{i=1}^{r} b_{i} s_{i j}+\sum_{k=2}^{l-1} c_{k j} z_{k} .
$$

Let us put $S^{\prime}=\left(d_{i j}\right)$. Multiplying $d_{j p}$ to the equation (11) and taking a sum on index $j$, we have

$$
z_{l} \sum_{j=1}^{r} a_{j} d_{j p}=s b_{p}+\sum_{j=1}^{r} \sum_{k=2}^{l-1} z_{k} c_{k j} d_{j p}
$$


for all $p=1, \cdots, r$. Since $\left(z_{1}=s, z_{2}, \cdots, z_{l}\right)$ is a regular sequence for $A$, there are elements $e_{j}, f_{k j} \in A(j=1, \cdots, r ; k=2, \cdots, l-1)$ such that

$$
\sum_{i=1}^{r} a_{i} d_{i j}=s e_{j}+\sum_{k=2}^{l-1} z_{k} f_{k j}
$$

for all $j$. On the other hand, we have $s \sum_{i=1}^{r} a_{i} X_{i}=\sum_{i, j=1}^{r} a_{i} d_{i j} Y_{j}$ since $S S^{\prime}=$ $S^{\prime} S=s I_{r}$. Hence it holds that

$$
s \sum_{i=1}^{r} a_{i} X_{i}=s \sum_{i=1}^{r} e_{i} Y_{i}+\sum_{i=1}^{r} \sum_{k=2}^{l-1} z_{k} f_{k i} Y_{i} .
$$

Since $\mathrm{A}$ is local and Noetherian, the sequence $\left(z_{2}, \cdots, z_{l-1}, s\right)$ is also a regular sequence for $A$. Hence we have

$$
\sum_{i=1}^{r} a_{i} X_{i} \in \sum_{i=1}^{r} A Y_{i}+\sum_{k=2}^{l-1} \sum_{i=1}^{r} A\left(z_{k} X_{i}\right)
$$

and the lemma is proved.

Proof of theorem, continued. By the lemma above, we can see $(Z, F)$ is ET-data for $E$. Finally, we show $\operatorname{elem}_{F}(E) \simeq \mathcal{O}_{X}^{r}$. Let $\left\{U_{i}\right\}_{i \in I}$ be an affine open covering of $X$ on which $E$ (resp : $\left.\operatorname{elem}_{F}(E)\right)$ has $G_{i j}\left(\right.$ resp : $H_{i j}$ ) as a transition matrix on $U_{i} \cap U_{j}$. By definitions of $G_{i j}$ and $S_{i}=\left.\left(s_{1}, \cdots, s_{r}\right)\right|_{U_{i}}$, it holds that ${ }^{t} S_{i}=G_{i j}{ }^{t} S_{j}$. On the other hand, from the exact sequence

$$
0 \rightarrow \operatorname{elem}_{F}(E) \rightarrow E \rightarrow F \rightarrow 0,
$$

we see that ${ }^{t} S_{i} H_{i j}=G_{i j}{ }^{t} S_{j}$ for all $i, j$. So $H_{i j}=I_{r}$, and this $\operatorname{implies~}_{\operatorname{elem}}(E) \simeq$ $\mathcal{O}_{X}^{r}$. Thus we can get the following commutative diagram:

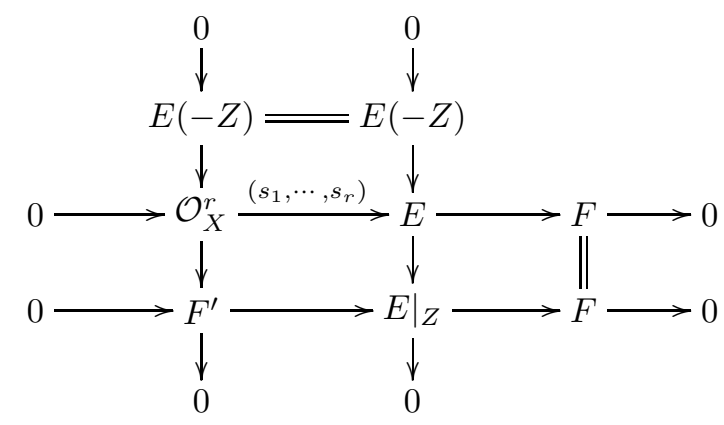

where $F^{\prime}:=\operatorname{ker}\left(\left.E\right|_{Z} \rightarrow F\right) \simeq \operatorname{coker}\left(E(-Z) \rightarrow \mathcal{O}_{X}^{r}\right)$. Note that $\operatorname{rank}_{Z} F=1$ and $\operatorname{rank}_{Z} F^{\prime}=r-1$. Also note that locally the morphism $E(-Z) \rightarrow \mathcal{O}_{X}^{r}$ in the first column of the above diagram is just the matrix $S^{\prime}$ which is adjoint to $S$. Combining this with Lemma 1.4, we can find the 1-ET-data $\left(Z, F^{\prime}\right)$ for $\mathcal{O}_{X}^{r}$ such that $\operatorname{elem}_{F^{\prime}}\left(\mathcal{O}_{X}^{r}\right) \simeq E(-Z)$; this completes the proof.

Let us compare the generalized elementary transformation with that defined by Maruyama. In Mar, Maruyama defined the elementary transformation of vector bundles by using the triple $(Z, F, \varphi)$, where $Z$ and $\varphi$ are the same as Definition 1.1. but where $F$ is not MCM but a vector bundle. In this case, we cannot apply the above argument on $\mathbf{P}_{\mathbb{K}}^{n}(n \geq 4)$, hence for example, we cannot construct the Horrocks-Mumford bundle (the Horrocks-Mumford bundle is an indecomposable 
2-bundle on $\mathbf{P}_{\mathbb{C}}^{4}$; see $[\mathrm{HN}$ for details) or Tango bundle (see Example (3) of this section, or [Ta-1] for details) from a trivial bundle. This is pointed out by Sumihiro in $[\mathrm{Su}-2$, and let us review it to see the difference between the two definitions.

At first, we must remember the Grothendieck-Lefschetz theorem. That implies if $Z$ is an effective divisor on $\mathbf{P}_{\mathbb{K}}^{n}$ (where $n \geq 4$ and $\mathbb{K}$ is an algebraically closed field of characteristic zero), then it holds that $\operatorname{Pic}\left(\mathbf{P}_{\mathbb{K}}^{n}\right) \simeq \operatorname{Pic}(Z) \simeq \mathbb{Z} \cdot \mathcal{O}_{Z}(1)$. Now, let us take the arbitrary triple $(Z, F, \varphi)$ for $\mathcal{O}_{\mathbf{P}_{\mathbb{K}}^{n}}^{2}(n \geq 4)$ of Maruyama's (i.e., $F$ is a line bundle). Then we can see that the elementary transformation $\operatorname{elem}_{F}\left(\mathcal{O}_{\mathbf{P}_{K}^{n}}^{2}\right)=: E$ by these data always splits. In fact, the Grothendieck-Lefschetz theorem implies $F \simeq \mathcal{O}_{Z}(d)$ for some integer $d \in \mathbb{Z}$. Considering the long exact sequence of the following short exact sequence induced by the elementary transformation

$$
0 \rightarrow E \rightarrow \mathcal{O}_{\mathbf{P}_{\mathbb{K}}^{n}}^{2} \rightarrow F \rightarrow 0,
$$

we can see that $H^{i}\left(\mathbf{P}_{\mathbb{K}}^{n}, E(l)\right)=0(\forall l \in \mathbb{Z}, i=1,2, \cdots, n-1)$. Hence by Horrocks' splitting criterion (for example, see Theorem 2.3.1 in [OSS]), this $E$ splits into the direct sum of line bundles. So to construct an indecomposable rank two vector bundle on the higher dimensional projective space, MCM sheaves on divisors are necessary.

At the end of this section, we see the geometric interpretation of the new elementary transformation and MCM sheaves. Let $X, Z, E, F$ be the same as in Definition 1.1. Consider the projective bundle $\pi: \mathbf{P}(E) \rightarrow X$ of rank $r-1$. Put $Y:=\mathbf{P}(F) \subset \mathbf{P}(E)$. Then $Y$ has some common properties for all MCM sheaves $F$. For example,

- $\pi(Y)=Z$.

- Locally, $Y$ is defined by the zero locus of $r$-linear equations.

Summarizing these properties of $Y$ combined with the result in [Su-2], we can find the geometric characterization of this elementary transformation.

Proposition 1.5. Let $X$ be a regular scheme, $E$ be a vector bundle of rank $r(>1)$, and $m$ be an integer such that $1 \leq m \leq r-1$. Then there is a one to one correspondence between the following two sets:

I) $(r-m)$-ET-data $(Z, F)$ for $E$.

II) The pair $(Z, Y)$, where $Z$ is a reduced divisor on $X$ and $Y$ is a closed subscheme of $\mathbf{P}(E)$ satisfying $\pi(Y)=Z$ by the canonical projection $\pi$ : $\mathbf{P}(E) \rightarrow X$. Moreover, for every $x \in Z$, there is an affine open neighborhood $U=\operatorname{Spec} A \subset X$ of $x$ which satisfies the following three conditions:

1) $\left.E\right|_{U} \simeq \oplus^{r} \mathcal{O}_{U}$,

2) let $s \in A$ be a local equation of $Z$ on $U$. Then in $\pi^{-1}(U) \simeq U \times \mathbf{P}^{r-1}$, $Y$ is defined by the following linear equations:

$$
s_{i 1} X_{1}+\cdots+s_{i r} X_{r}=0(i=1,2, \cdots, r)
$$

where $S:=\left\{s_{i j}\right\}_{i, j=1}^{r} \in M(r, A)$ and $X_{1}, \cdots, X_{r}$ are homogeneous coordinates of $\mathbf{P}^{r-1}$, and

3) the rank of $S$ at every generic point of $Z$ is $m$. Moreover, there exists a matrix $S^{\prime} \in M(r, A)$ such that $S S^{\prime}=S^{\prime} S=s I_{r}$ and the rank of $S^{\prime}$ at every generic point of $Z$ is $r-m$.

Proof. We construct the maps between I) and II), which induce the one to one correspondence between them. From I) to II), it is sufficient to put $\mathbf{P}(F)=: Y \subset$ 
$\mathbf{P}(E)$ and check properties 1), 2), and 3) above. From II) to I), we use the surjection

$$
L_{E} \rightarrow \mathcal{O}_{Y} \otimes L_{E} \rightarrow 0
$$

where $L_{E}$ is a tautological line bundle on $\mathbf{P}(E)$. Pushing forward this sequence by $\pi$ and taking the image, we have the new surjection

$$
E \rightarrow F \rightarrow 0 .
$$

By using Lemma 1.4, we can check that $(Z, F)$ is ET-data for $E$. It is easy to see they give the one to one correspondence.

This proposition tells us that the new definition shows the relation between Maruyama's and Sumihiro's definition. Note also that from this proposition, Theorem 1.3 corresponds to Theorem $3.2 \mathrm{in}[\mathrm{Su}-2]$.

\section{Elementary transformation of Reflexive sheaves}

In this section, we apply the slight modified result of section one to reflexive sheaves. Through the "elementary transformation of reflexive sheaves", we can obtain the similar results to that of vector bundles, and we can see the relation between these two categories of sheaves. Before the discussion, we review some definitions and results of reflexive sheaves. All definitions, results, and proofs can be seen in [H3].

Definition 2.1. A coherent sheaf $E$ on an integral scheme $X$ is reflexive if the canonical morphism $E \rightarrow E^{* *}$ is an isomorphism.

By the definition, vector bundles are reflexive, and reflexive sheaves are torsion free. In this section, we mainly use the following remarkable properties of reflexive sheaves.

Proposition 2.1 ([H3], Corollary 1.4). $\operatorname{codim}_{X}(\operatorname{Sing}(E)) \geq 3$ for a reflexive sheaf $E$ on a regular scheme $X$.

Proposition 2.2 ([ [H3], Corollary 1.5). Let $X$ be a normal scheme, and let

$$
0 \rightarrow E^{\prime} \rightarrow E \rightarrow F \rightarrow 0
$$

be an exact sequence of coherent sheaves on $X$, with $E$ reflexive. Then $E^{\prime}$ is reflexive if and only if $\operatorname{Ass}_{X}(F)$ consists of points whose codimensions are 0 or 1.

Proposition 2.3 ([ $[\mathrm{H} 3$, Proposition 1.6). For a coherent sheaf $E$ on a normal scheme $X$, the following conditions are equivalent:

1) $E$ is reflexive.

2) $E$ is torsion free and normal.

3) $E$ is torsion free, and for each open set $U \subset X$ and each closed set $Z$ in $U$ satisfying $\operatorname{codim}_{U}(Z) \geq 2$, we have $\left.E\right|_{U} \simeq j_{*}\left(\left.E\right|_{U \backslash Z}\right)$, where $j: U \backslash Z \rightarrow U$ is an open immersion.

Now, we can define an elementary transformation of reflexive sheaves on regular schemes. At first, we prepare the ET-data for reflexive sheaves.

Definition 2.2. Let $X$ be a regular scheme, $r(\geq 2)$ and $m$ be an integers such that $1 \leq m \leq r-1$, and $E$ be a reflexive sheaf of rank $r$ on $X$. Then we say the 
triple $(Z, F, \varphi)$ is $m$-weak-elementary-transformation-data for $E$ ( $m$-w-ET-data for $E$, for short) if the following three conditions are satisfied:

1) $Z$ is an effective reduced divisor on $X$,

2) $F$ is a torsion free $\mathcal{O}_{Z}$-module of rank $r-m$, and

3) $\varphi$ is a surjection from $E$ to $F$ as $\mathcal{O}_{X}$-modules.

We usually denote w-ET-data $(Z, F, \varphi)$ by $(Z, F)$ if there is no confusion. By the same way as vector bundles, the elementary transformation of reflexive sheaves can be defined as follows.

Definition 2.3. With the notation in Definition 2.2, we denote the kernel of $\varphi$ : $E \rightarrow F$ by $\operatorname{elem}_{F}(E)$ and call it the elementary transformation of $E$ by w-ET-data $(Z, F)$.

The next lemma tells us why we call this definition an elementary transformation of reflexive sheaves.

Lemma 2.4. With the above notation, $\operatorname{elem}_{F}(E)$ is a reflexive sheaf of rank $r$.

Proof. Note that $\operatorname{Ass}_{X}(F)$ consists of the generic points of $Z$.

The next theorem is the main result of this section, which is the reflexive version of Theorem 1.3 .

Theorem 2.5. Let $X$ be a nonsingular quasi-projective variety over an algebraically closed field $\mathbb{K}, \mathcal{O}_{X}(1)$ be an ample line bundle on $X$, and $E$ be a reflexive sheaf of rank $r(>1)$ on $X$. Then there exist 1-w-ET-data $(Z, F)$ for $\mathcal{O}_{X}^{r}$ such that $Z$ is normal and $\operatorname{elem}_{F}\left(\mathcal{O}_{X}^{r}\right) \simeq E \otimes L$ for some line bundle $L \in \operatorname{Pic}(X)$. Moreover, when $\operatorname{dim} X \geq 2$, we can take $Z$ as an integral normal divisor.

Proof. From Proposition 2.1, there is a nonempty open set $U \subset X$ such that $\operatorname{codim}_{X}(X \backslash U) \geq 3$ and $\left.E\right|_{U}$ is locally free. Since $U$ is nonsingular and quasiprojective, we can apply the argument in the proof of Theorem 1.3 to the vector bundle $\left.E\right|_{U}$ on $U$, and we obtain the ET-data $(Z \cap U, F)$ on $U$. Note that by Proposition 2.1. we only have to consider the case when $\operatorname{dim} X \geq 3$ (otherwise $E$ is automatically locally free, so the proof is reduced to that of Theorem 1.3). Since all the points belonging to $Z \backslash U$ are of codimension $\geq 2$ in $Z$, we can also use Serre's criterion. From the fact that $Z \cap U$ is integral and normal, we can see that $Z$ is also integral and normal. Note the following two facts:

(1) If we put $j: U \rightarrow X$ as an open immersion, then $j_{*}\left(\left.E\right|_{U}\right) \simeq E$, and

(2) the direct image $j_{*}(F)$ of MCM sheaves $F$ on $Z \cap U$ which have a surjection from some vector bundle on $U$ is a torsion free $\mathcal{O}_{Z}$-module on $Z$.

(1) follows immediately from the third condition of Proposition 2.3 and (2) follows from the property of direct images. Combining these facts with the proof of Theorem 1.3, the proof is completed.

\section{ACKNOWLEDGEMENT}

The author is grateful to Professor Masaki Maruyama for much advice and support. The author also thanks many friends who often kindly helped him. The author is so grateful to the referee for the careful reading of the first edition of this article, as well as much advice and suggestions. 


\section{REFERENCES}

[A] T. Abe, The elementary Transformation of vector bundles on regular schemes, preprint in Kyoto University, 9, 2004.

[AY] T. Abe and M. Yoshinaga, Splitting criterion for reflexive sheaves, arXiv, mathAG/0503710, preprint in RIMS, 1496 (2005).

[DK] I. Dolgachev and M. Kapranov, Arrangements of Hyperplanes and Vector Bundles on $\mathbf{P}^{n}$, Duke. Math. J., 71 (1993), 633-664. MR1240599 (95e:14029)

[G] A. Grothendieck, Local cohomology, Lecture Notes in Math., 41, 1967. MR0224620 $(37: 219)$

[H1] R. Hartshorne, Ample Subvarieties of Algebraic Varieties, Lecture Notes in Math., 156, Springer-Verlag, 1970. MR0282977 (44:211)

[H2] R. Hartshorne, Algebraic Geometry, Graduated Texts in Mathematics, Springer-Verlag, 1977. MR0463157 (57:3116)

[H3] R. Hartshorne, Stable reflexive sheaves, Math. Ann. 254 (1980), 121-176. MR0597077 (82b:14011)

[HN] G. Horrocks and D. Mumford, A rank 2 vector bundle on $\mathbf{P}^{4}$ with 15,000 symmetries, Topology. 12 (1973), 63-81. MR0382279 (52:3164)

[K] G. Kempf, A criterion for the splitting of a vector bundle, Forum Math. 2 (1990), 477-480. MR.1067213 (91j:14036)

[LY] H. S. Luk and S. T. Yau, Cohomology and splitting criterion for holomorphic vector bundles on $\mathbb{C P}^{n}$, Math. Nachr. 161 (1993), 233-238. MR.1251020 (94k:14037)

[Mar] M. Maruyama, On a family of algebraic vector bundles, Number Theory, Algebraic Geometry and Commutative Algebra (1973), 95-149, Kinokuniya. MR0360587 (50:13035)

[Mat] H. Matsumura, Commutative Algebra, W.A. Benjamin Co., New York (1970). MR0266911 $(42: 1813)$

[OSS] C. Okonek, M. Schneider and H. Spindler, Vector Bundles on Complex Projective Spaces. 3 (1980), Birkhäuser. MR0561910 (81b:14001)

[Sa] K. Saito, Theory of logarithmic differential forms and logarithmic vector fields, J. Fac. Sci. Univ. Tokyo Sect. IA Math 27 (1980), no. 2, 265-291. MR0586450 (83h:32023)

[Sch] C. Schoen, On the geometry of a special determinantal hypersurface associated to the Mumford-Horrocks vector bundle, J. reine. angew. Math. 364 (1986), 85-111. MR0817640 (87e:14039)

[Su-1] H. Sumihiro, A theorem on splitting of algebraic vector bundles and its applications, Hiroshima Math J. 12 (1982), 435-452. MR0665505 (83m:14010)

[Su-2] H. Sumihiro, Elementary Transformations of Algebraic Vector Bundles, Algebraic and Topological Theories (1985), 305-327, Kinokuniya. MR1102263 (92e:14012)

[Su-3] H. Sumihiro, Elementary Transformations of Algebraic Vector Bundles II, Algebraic Geometry and Commutative Algebra in honor of Masayoshi NAGATA (1987), 713-748. MR0977780 (92e:14013)

[Su-4] H. Sumihiro, Determinantal varieties associated to rank two vector bundles on projective spaces and splitting theorems, Hiroshima Math. J. 29 (1999), 371-434. MR1704256 (2000f:14077)

[ST] H. Sumihiro and S. Tagami, A splitting theorem for rank two vector bundles on projective spaces in positive characteristic, Hiroshima Math J. 31 (2001), 51-57. MR1820694 (2001m:14063)

[Ta-1] H. Tango, On morphisms from projective space $\mathbf{P}^{n}$ to the Grassmann variety $G r(n, d), \mathrm{J}$. Math. Kyoto Univ. 16-1 (1976), 201-207 MR0401787 (53:5614)

[Ta-2] H. Tango, On Vector Bundles on $\mathbf{P}^{n}$ Which Have $\sigma$-Transition Matrices, Tokyo J. Math. 16-1 (1993), 1-29. MR1223285 (94d:14019)

Department of Mathematics, Graduate School of Science, Kyoto University, Kyoto, 606-8502, JAPAN

E-mail address: abetaku@kusm.kyoto-u.ac.jp

Current address: Department of Mathematics, Hokkaido University, Kita-10, Nishi-8, Kita-Ku, Sapporo, Hokkaido, 060-0810, Japan

E-mail address: abetaku@math.sci.hokudai.ac.jp 